\section{$\underset{\substack{\text { hommes } \\ \text { \& migrations }}}{ }$}

\section{Hommes \& migrations}

Revue française de référence sur les dynamiques

migratoires

$1285 \mid 2010$

L'appel du pied

\title{
Voyage au bout de la mer : les boat people en
}

\section{France}

\section{Martine Gayral-Taminh}

\section{OpenEdition \\ Journals}

Édition électronique

URL : http://journals.openedition.org/hommesmigrations/1200

DOI : $10.4000 /$ hommesmigrations. 1200

ISSN : 2262-3353

Éditeur

Musée national de l'histoire de l'immigration

\section{Édition imprimée}

Date de publication : 1 mai 2010

Pagination : 163-171

ISSN : 1142-852X

\section{Référence électronique}

Martine Gayral-Taminh, « Voyage au bout de la mer : les boat people en France », Hommes \& migrations [En ligne], 1285 | 2010, mis en ligne le 29 mai 2013, consulté le 22 septembre 2020. URL : http:// journals.openedition.org/hommesmigrations/1200 ; DOI : https://doi.org/10.4000/

hommesmigrations. 1200

Ce document a été généré automatiquement le 22 septembre 2020.

Tous droits réservés 


\title{
Voyage au bout de la mer : les boat people en France
}

\author{
Martine Gayral-Taminh
}

1 Avant 1975, peu de Vietnamiens quittaient leur pays pour des raisons économiques et de façon définitive. L'émigration des Vietnamiens en France était essentiellement le fait d'étudiants qui rentraient au pays à la fin de leurs études. Après le départ des Français en 1956, l'aggravation de la guerre a contraint des étudiants, plus souvent originaires du Sud, à prolonger leur séjour et à s'établir en France. Le changement politique radical du "Vietnam du Sud", qui accompagne la fin de la guerre du Vietnam (avril 1975) et l'unification du pays sous l'autorité du Nord communiste (officiellement acquise le 2 juillet 1976), provoque une vague d'émigration vers la France et différents pays d'Europe ou d'Amérique du Nord et vers l'Australie.

2 De façon proprement tragique, ce n'est ni à l'ancestral ennemi chinois, ni au colonisateur français, ni aux désastres de la surpuissante armée américaine, mais au frère du Nord que le Sud doit de connaître le pire exode de son histoire, un exil en France de plus de 100000 personnes, phénomène unique dans toute l'histoire du Vietnam $^{1}$. L'installation du nouveau pouvoir politique ne rencontre pas au Sud de réelle opposition et se fait sans véritable violence. Confrontés à de graves difficultés, notamment économiques $\left({ }^{2}\right)$, les dirigeants laissent d'abord filer à l'étranger ceux qui le désirent.

Il s'agit le plus souvent d'immigration familiale. Toute une parentèle, fratrie et surtout parents âgés, fuyant le nouveau régime, rejoint la fraction de la famille émigrée avant elle. Avec l'ouverture politique du pays dans les années quatre-vingt-dix, certains, parmi les rares qui ont conservé là-bas des parents et leur maison familiale, retournent définitivement au pays. “Ils aimeraient mourir là-bas". D’autres s'essaient à pratiquer pour un temps l'aller-retour. Beaucoup vieillissent auprès de leurs enfants et petitsenfants français. Les plus nombreux sont morts aujourd'hui. Tous sont restés inconnus des services sociaux et de la société française.

4 Puis, débordés au tournant des années quatre-vingt par la contestation sociale et par les demandes d'autorisation de quitter le pays qui affluent comme autant de désaveux, 
le pouvoir répond par la fermeture des frontières et la répression policière. Une sorte de folie collective s'empare alors de franges entières de la population qui se jettent à la mer, prêtes à tout pour fuir. Suivant une stratégie.

Des motivations économiques et familiales

6 Le récit de vie de trois de ces immigrés vietnamiens venus en France au milieu des années soixante-dix, un homme et deux femmes, ne prétend pas rendre compte de la diversité des situations. Au travers de ce que leur parole nous apprend de leurs trajectoires d'immigration, des circonstances et des motifs de leur venue, des parcours de leur installation, de leur histoire en France, se dessine un modèle d'immigration.

7 Madame Li appartient à la première vague d'immigration. Tout juste 60 ans aujourd'hui, après avoir partagé son enfance entre Phnom Penh et Saigon, son mariage unit deux familles de commerçants sino-vietnamiens. Alarmé par des difficultés professionnelles, mais aussi par le climat général de guerre larvée, le couple anticipe l'avenir et gagne sans peine la France en 1973, accompagné de leur jeune enfant. Madame Li avait 24 ans. "Je suis venue par mes propres moyens, j'ai acheté le passeport, le billet d'avion." Prenant appui sur le réseau familial en place, trois frères de son mari venus poursuivre des études en France et installés depuis lors, elle ouvrira à son tour la porte de l'immigration à ses sœurs dont elle se portera caution. Elle est ainsi partie prenante d'une stratégie familiale d'immigration qui réussit, demeurant en cela fidèle au modèle asiatique sino-vietnamien, aux valeurs confucéennes de la famille et du travail.

8 La communauté familiale unit ses forces pour mettre en valeur un restaurant. "On n'avait pas de maison, on mangeait au restaurant, on dormait au restaurant". Deux ans plus tard, le couple monte sa propre affaire. Face à des débuts difficiles, leur travail acharné laisse peu de place aux enfants et aux considérations de santé. Le succès vient enfin avec la nouvelle clientèle des boat people. "On travaillait, on ne demandait pas de sous. Je n'aime pas les gens qui demandent des sous." On trouve là non seulement l'expression d'une fierté d'un travail bien fait et reconnu comme tel, mais aussi la revendication d'une autonomie qui ne doit rien à personne, qui ignore l'aide sociale. Ces immigrés asiatiques qui participent ainsi fortement à l'économie locale, à la création d'emplois, même si c'est sur le mode de l'entre-soi et qui paient des impôts, par cette forme de citoyenneté concourent à la représentation positive de l'immigré vietnamien.

Déployée au bénéfice de trois enfants, la réussite économique du couple est aussi celle du projet parental, celle de l'ascension sociale par les études et l'activité professionnelle. Le fils aîné, âgé de 21 ans, effectue aujourd'hui "un stage à la banque au Japon" et le plus jeune poursuit des études à Paris, tous les deux dans le cadre d'écoles de commerce.

L'inscription sociale de Madame Li par le travail ne se double cependant d'aucune inscription relationnelle : "Je n'ai pas d'amis, ni français, ni... J'ai des clients, des relations." En revanche, l'attitude d'un mari entreprenant et généreux qui finance, ici, en France, des associations vietnamiennes préoccupées de développement pour le Vietnam, donne lieu à une reconnaissance sociale de la part de la communauté vietnamienne locale. L'inscription sociale de Madame Li se fait en France par des voies transgénérationnelles et d'alliance familiale. Limitée jusqu'alors dans sa pratique à des intérêts commerciaux, elle ne parle que médiocrement le français. Sa fille ayant épousé un Français, elle souhaite l'améliorer, car ses deux petites filles sont françaises. 
11 Madame Li n'a plus de liens avec le pays de son enfance où elle ne se rend qu'à des fins purement touristiques. Reste la France, où, au fil de sa vie, se sont développées ses véritables racines: "Je n'ai que des souvenirs en France [...] et j'aime la France". Ignorant toute forme de revendication identitaire, elle n'a cependant rien cédé des fondamentaux de la culture traditionnelle qu'elle a transmise à ses enfants, reproduisant ainsi le modèle reçu de ses propres parents: "La valeur du travail et le respect des ancêtres."

12 Chinoise vietnamienne par la morale traditionnelle qui l'anime, elle est définitivement française par son vécu : "En France j'ai connu le malheur, le bonheur. Mes enfants, mes petits-enfants sont en France. Maintenant j'ai la maison, j'ai l'argent, tout s'est fait avec la France. C'est ma vie la France." L'alliance se dit ici sans réserve.

\section{Les boat people à la recherche d'une vie meilleure}

13 Chaque famille du Sud peut faire état d'un boat people. La recherche d'un meilleur niveau de vie, l'aspiration à la liberté et à l'instruction pour les enfants sont déterminants dans la décision de partir. Le voyage conduit les plus nombreux dans les camps gérés par le HCR avant d'être accueillis par un pays occidental. Face aux difficultés rencontrées dans la gestion de camps surpeuplés, les pays frontaliers mettent en place, à la fin des années quatre-vingt, des mesures de restriction de l'accueil. De telles mesures s'accompagnent d'un ralentissement du flux, mais c'est avec le développement économique et l'ouverture politique du Vietnam que le mouvement s'éteint de lui-même.

14 Lam témoigne pour tous ceux dont la tentative de départ a échoué. Né dans une riche famille de commerçant, devenu professeur de philosophie, il est victime de la discrimination politique exercée à l'encontre des intellectuels. "En 1975, tous les professeurs ont été mis dehors, sauf les professeurs de maths." Il survit un temps de petits boulots : "On vendait du café, des pelles, on faisait un peu de tout." Mais la disqualification sociale, la restriction des libertés ainsi que le manque de perspectives familiales vont avoir raison de lui et le poussent à contrecœur à quitter le Vietnam. "Les enfants sont la vraie raison du départ. Il fallait qu'ils puissent étudier."

15 Mesurant le danger de l'entreprise - "sur deux millions de partis, seulement la moitié est arrivée" -, la famille se scinde. Âgé alors de 37 ans, marié et père de trois enfants, attendu en France par une de ses sœurs, Lam tente sa chance avec les deux aînés. Seuls les enfants réussissent. Lui paie son échec de 10 mois de rétention dans les prisons vietnamiennes. La famille ne se reconstitue en France que neuf ans plus tard. "Nous sommes venus ensemble, ma femme et ma fille, en 1990 comme réfugiées politiques". L'installation s'effectue alors sur le modèle de tous les demandeurs d'asile : accueil en CADA, aide à la recherche d'un emploi et d'un logement indépendant, aide alimentaire et pécule de soutien, etc. Toute la famille obtient sans délai la nationalité française.

Lam témoigne d'efforts certains pour gagner son autonomie. "J'ai fait un peu de bricolage, j'ai donné des cours de guitare. [...] J'ai commencé par travailler dans un restaurant, mais après un an il a déposé le bilan. Puis j'ai travaillé à la chaîne dans une entreprise fabriquant des machines à couper l'herbe, un travail temporaire". Efforts partagés par sa femme: "Elle a travaillé deux ans dans une école primaire, là-bas elle était sage-femme." Dans l'impossibilité de trouver un travail stable et suffisamment rémunéré, le couple se lance au bout de 
deux ans dans un commerce de restaurant-traiteur sur le modèle de nombreux compatriotes. Mais le succès se fait attendre.

\section{Du déclassement professionnel au déracinement}

17 Lam n'en fait pas moins que les autres, mais il ne s'en sort pas. Tombé dès son arrivée dans l'aide sociale, il la sollicite pour tout : des papiers d'abord, puis des vêtements, des denrées alimentaires. "Une assistante sociale m'a cherché une maison". Il ne se départira jamais du statut d'assisté et s'en réclame encore aujourd'hui pour sauver son entreprise de la faillite. Une telle situation, en contradiction avec la revendication d'autonomie que nourrissent la plupart de ses compatriotes, le coupe de ses pairs, faisant de lui un homme isolé, privé de vie sociale. La non-équivalence des diplômes vietnamiens est directement responsable du déclassement professionnel de Lam. Sachant le discrédit dont souffre le travail manuel dans la tradition vietnamienne qui classe au premier rang les lettrés, au second les paysans et artisans, au dernier les commerçants $\left({ }^{3}\right)$; sachant aussi le rang que se flattait d'avoir atteint Lam, fils d'un riche commerçant, nanti par la suite de diplômes et exerçant une profession d'intellectuel, on imagine sans peine le sentiment de disqualification qui est aujourd'hui le sien. Et quand l'ouverture d'un commerce ethnique assure pour d'autres l'autonomie et l'insertion économique de la famille, semblable démarche constitue pour lui un nouvel échec. La situation financière et le statut social de Lam ne sont aujourd'hui guère supérieurs à ceux qu'il connaissait au Vietnam avant son départ. "En venant ici, ma femme et moi, nous sommes devenus des zéros." Pourtant, fidèle à la tradition vietnamienne qui privilégie la réussite scolaire des enfants, il a en cela rempli son contrat parental. Ses trois enfants ont réussi leurs études et exercent des professions intellectuelles. Lam trouve là quelques raisons de réconfort. Tous les siens se sont engagés dans l'occidentalisation. Chacun des enfants a fondé une famille. L'aînée est mariée avec un Vietnamien. Les deux autres ont épousé un ou une Français(e) et ont chacun un enfant. Aucun d'eux n'envisage de quitter la France. "Seul mon fils est venu avec moi là-bas mais il n'aime pas. [...] Ma femme non plus ne veut plus retourner là-bas".

Voué à un mal-être permanent, âgé de 64 ans aujourd'hui et à la retraite depuis peu, Lam n'aspire plus qu'à rentrer chez lui, au Vietnam, et à retrouver sa maison familiale. "J'ai une villa là-bas, je vis toujours avec mes souvenirs, je pense que je vais retourner dans deux ou trois ans au Vietnam", seul s'il le faut, "tout le monde est enchanté d'être français. [...] Moi, je ne suis français que par les papiers, mais pas dans la tête".

\section{Reconquérir la liberté d'entreprendre}

Dans le même contexte historique, la conduite du projet d'immigration de Em est tout autre. Comme son mari, elle appartient à la classe populaire des petits commerçants du delta du Mékong. Sa décision résulte des difficultés rencontrées dans son travail suite à l'interdiction d'exercer toutes formes de commerce, en l'occurrence pour elle celui du poisson. En 1979, à 28 ans, Em ouvre avec son mari la voie de l'immigration familiale, confiant sa petite fille aux soins de sa belle-mère. Une séparation de six ans. Em ne fait pas mystère sur les conditions du départ et du voyage qui conduit le couple dans un camp de réfugiés en Malaisie où il attend 12 mois la décision française de l'accueillir ${ }^{4}$. Le moment venu, le HCR organise son départ vers la France où Em rejoint son oncle, un 
ancien combattant. Avec le statut de réfugié, le couple s'inscrit dans une installation définitive. "J'avais la carte de réfugié, maintenant je suis française."

D'emblée, le couple manifeste des réserves vis-à-vis de l'assistance auquel leur donne droit leur statut. En quête d'autonomie - ils remboursent leur voyage en avion - le couple souhaite limiter au minimum le temps de l'assistance. "Après trois mois dans le foyer pour apprendre un petit peu de français, et préparer les papiers, j'ai demandé un travail intérim et j'ai commencé dans les usines X".

Em enchaîne en effet les travaux d'ouvrière postée, les horaires en 3x8, etc. "Toutes les choses que je pouvais faire." Au même moment, son mari travaille tour à tour comme manutentionnaire dans une entreprise, puis comme ouvrier dans un atelier de couture, avant d'être licencié pour raisons économiques. Peinant à obtenir un emploi salarié durable, le couple ouvre un petit restaurant de 28 couverts, empruntant ici sa démarche au modèle vietnamien des "familles confrontées à des difficultés d'intégration professionnelle [...] qui ouvrent des restaurants de taille réduite, avec une organisation de type familial et un fonctionnement artisanal". L'acquisition du restaurant se fait au prix de beaucoup de travail, de quinze ans de bonne gestion de vie et d'économie. Sa vente toute récente, puis la location des murs assurent la retraite du couple dans le cadre d'une vie simple et indépendante. "Nous, Asiatiques, on ne demande pas beaucoup de choses ". Une réflexion qui témoigne de l'engagement bouddhiste du couple. La vie sociale de Em se partage ici entre des relations françaises dans le cadre d'activités sportives et des relations avec ses compatriotes dans le cadre d'activités religieuses (bouddhistes) et associatives tournées vers le Vietnam. Et, à la faveur de fréquents séjours au Vietnam, elle entretient des liens de solidarité ordinaires avec ses frères et sœurs restés sur place.

22 Le projet d'immigration tel que le réalise Em demeure fidèle au modèle de la tradition vietnamienne qui valorise la réalisation individuelle par le travail et le rôle social d'une éducation parentale centrée sur l'instruction. À ce titre, Em a privilégié la réussite scolaire, pilier majeur de la promotion sociale de la famille confucéenne, d'autant que ni elle ni son mari n'ont suivi une scolarité qualifiante. Sa fille unique vit aux États-Unis avec "un Chinois de Taïwan. Elle a fini ses études et trouvé du travail dans le commerce international". L'ascenseur social par les études a bien fonctionné, lui ouvrant les voies de l'intégration au modèle sociétal occidental qui dépasse en l'occurrence les frontières françaises. Il a, ce faisant, fonctionné pour la famille entière.

C'est dans le respect de telles valeurs que Em a accompli une ascension sociale et acquis la reconnaissance de sa communauté d'origine, au Vietnam aussi bien qu'ici. Statut et reconnaissance sociale paraissent en l'occurrence bien supérieurs à ceux qu'elle aurait pu attendre en dehors de l'immigration.

\section{Une immigration qui fait souche}

Ces trois récits décrivent tous une immigration familiale qui déplace plusieurs générations et s'appuie sur le réseau familial et ethnique en place. Ils soulignent le rôle déterminant que jouent les motivations et l'engagement déployés au service d'un projet dont le véritable enjeu est l'intégration dans le pays d'accueil. Pour aucun d'eux, plus généralement pour la plupart des boat people, quelles que soient les conditions dramatiques du départ, le motif ne répond pas aux critères définis par la Convention de 
Genève. Deux des récits arguent de raisons strictement économiques et le dernier invoque le regroupement familial. des commerçantes Madame Li et Em sont exemplaires. En dépit de conditions de départ bien différentes $\left.{ }^{8}\right)$, toutes deux partagent la même détermination dans la réalisation d'un projet d'installation définitive. La réalisation d'un tel projet se déroule en deux temps, sur deux générations. L'enjeu à court terme est la réussite professionnelle des parents. Celle-ci, chez Madame Li, prend appui sur le réseau familial ; chez Em, sur les aides offertes aux réfugiés. Pour toutes deux, il s'agit là d'une étape obligée, mais de courte durée, limitée à l'accès au travail, à des conditions économiques qui assurent l'autonomie et permettent d'engager l'ascension sociale, en même temps que l'exercice d'un métier et la construction d'une famille, une famille aux "normes" traditionnelles s'entend. Il incombe à la seconde génération de franchir la seconde étape, par là d'assurer la promotion sociale de chacun des membres de la famille et celle de la famille tout entière. Initiée par une scolarité exemplaire (exigée telle par les parents), poursuivie par des études supérieures (ici demeurées inaccessibles aux parents) donnant accès à des professions intellectuelles, cette seconde étape constitue l'étape ultime du projet d'immigration. Un tel défi est à la portée de Madame Li et de Em. Reste sa mise en œuvre qui ne soulève aucune hésitation de leur part, quel que soit le prix à payer. Et c'est dans le succès de l'entreprise familiale, toujours conformément aux normes traditionnelles d'inspiration confucéenne, que l'immigration prend tout son sens, que gratification et fierté sont au rendez-vous.

On observe dans le cas de Lam une situation, certes plus complexe, mais comparable par certains points. Le sens pour lui d'une migration reportée de dix ans demeure incertain. Nanti de diplômes non reconnus en France, il demeure dans l'incapacité d'acquérir son autonomie et reste condamné à vie à l'aide sociale, par là disqualifié aux yeux de ses pairs. Absents au Vietnam, les dispositifs institutionnels de l'assistance sont inconnus des Vietnamiens. Le recours à de telles pratiques sont inconciliables avec le code de bonne conduite confucéen où travail et études constituent le socle de la construction personnelle et de la promotion sociale. En dépit du malaise que la plupart des Vietnamiens expriment dans leur rapport à l'aide sociale, cette aide a accompagné l'installation de certains mais est restée temporaire. Les assistantes sociales se plaisent à dire qu'il n'existe pas ou peu de Vietnamiens dans leurs dossiers' ${ }^{9}$.

Lam vit le drame de celui qui, faute de réussite dans son travail, doit renoncer à tout espoir d'ascension économique, et plus encore à la promotion sociale, seule susceptible de mettre fin à la disqualification personnelle, familiale et sociale qui est la sienne et celle de sa famille tout entière, depuis la perte de son poste d'enseignant au Vietnam. 
Sa trajectoire d'immigration ne trouve sens qu'à la faveur de deux médiations, l'une matérielle, l'autre intellectuelle, assurées toutes deux par la génération des enfants. C'est en effet par l'intermédiaire de ses propres enfants, à travers leurs études, puis à travers leur réussite professionnelle, marquée par l'accès à des professions intellectuelles, à travers enfin les mariages et naissances que Lam renoue en quelque façon avec la richesse, résultat du travail, signe de la dignité. Il renoue aussi avec le savoir savant, indice de la supériorité intellectuelle et morale dans la hiérarchie confucéenne, il retrouve enfin la voie du succès et de la promotion sociale familiale. Une voie en tout point conforme à la doctrine. En cela, Lam, bon père, sauve la face. d'une bonne insertion dans le tissu économique et professionnel en continuant d'emprunter aux conseils de maître Khong $\mathrm{Tu}$. Un processus d'intégration qui s'ancre dans la culture d'origine ; une sphère privée où langue et cuisine vietnamienne sont la règle et où, dans chaque maison, l'autel des ancêtres témoigne de la fidélité au culte. Autant de signes d'un fort attachement à la culture d'origine. Et si intégration rime avec perte de la culture d'origine, alors, certainement non, les Vietnamiens considérés dans cette étude ne sont pas intégrés. Pourtant, c'est à travers la voie offerte à leurs enfants que cette génération participe à l'intégration "totale", voire l'acculturation progressive de cette nouvelle lignée d'immigrés vietnamiens. C'est ce vers quoi s'acheminent les générations suivantes, notamment à la faveur de mariages mixtes, bien acceptés ${ }^{13}$. Un tel processus d'intégration-acculturation est la réponse paradoxale qu'apportent ces immigrés restés fidèles à leur culture vietnamienne. "La morale 
confucéenne apparait bien vivace dans leur esprit et se révèle une des clés de leur intégration. [...] 'Dans un ballon on s'arrondit, dans un tube on s'étire', 'o bau thi tron, o ong thi dai'14".

\section{BIBLIOGRAPHIE}

Fourgeau Catherine, "L'insertion en France des communautés asiatiques", Migrations Études, n 80, avril-mai, 1998, pp. 1-13.

Gayral-Taminh Martine, "Une immigration invisible, gage d'intégration ? Récits de vie d'étudiants vietnamiens émigrés en France dans les années 1955-1970”, Ethnologie française, XXXIX (4), 2009, pp. 721-732.

Raullin Anne, L'ethnique est quotidien, Paris, L'Harmattan, coll. “Connaissance des hommes”, 2000. Bergeret Pascale, Paysans, État et Marchés au Vietnam. Deux ans de coopération agricole dans le bassin du Fleuve Rouge, Paris, Karthala. coll. "Hommes et Sociétés : Sciences économiques et politiques", 2002

Le Huu Khoa, "L'immigration asiatique : espaces communautaires et stratégies d'ascension professionnelle", Migrations Études, n 56, mars-avril 1195, pp. 1-12.

Le Huu Khoa, "Femmes asiatiques en France. Places familiales, placements professionnels et déplacements sociaux", Migrations Études, n 134, mars 2006, pp. 1-12.

Simon-Barouh Ida, "Les Vietnamiens en France", in Hommes et Migrations, Hors-Dossier, n 1219 , 1999, pp. 69-89.

Morillon Anne, "Les réfugiés politiques face à la naturalisation", Hommes et Migrations, $\mathrm{n}^{\circ} 1234$, 2001, pp. 50-57.

Schnapper Dominique, Qu'est-ce que l'intégration ?, Paris, Gallimard, coll. Folio Actuel, 2007.

Rafaï Kadija, Mantovani Jean, Duchier Jenny, Gayral-Taminh Martine, Le vieillissement des migrants. Situation en Midi-Pyrénées, rapport ORSMIP, octobre 2007.

Le Huu Khoa, L'immigration confucéenne en France. On s'exile toujours avec ses ancêtres, Paris, l'Harmattan, 1996.

Vidal Mai Lan, “L’immigration à la vietnamienne”, Esprit, mai 1997, pp. 171-178.

\section{NOTES}

1. Entre 1975 et 1989, 122?093 Vietnamiens auraient été accueillis en France au titre officiel de "réfugiés". D'après un responsable de l'ofpra, ils seraient aussi nombreux sur la période à s'être introduits en France selon d'autres procédures

2. Ils ont à faire face à des situations de famine résultant des destructions de la guerre, de l'arrêt de l'aide soviétique, de la gestion catastrophique des ressources sur le modèle collectiviste soviétique et de la faible collaboration des gens du Sud, grenier à riz du pays. 
3. Simon-Barouh Ida, "Les Vietnamiens en France", in Hommes et Migrations, Hors-Dossier, $\mathrm{n}^{\circ}$ 1219, 1999, pp. 69-89.

4. Tempêtes, attaques, vols et viols par des pirates et des policiers thaïlandais. Dans un bateau surpeuplé, sans moteur et à la dérive, ils doivent leur salut à des pêcheurs de Thaillande qui les remorquent à proximité de l'île du camp qu'ils rejoignent à la nage pour échapper à la police malaise chargée de les refouler à la mer.

5. Le Huu Khoa, "L'immigration asiatique: espaces communautaires et stratégies d'ascension professionnelle", op. cit.

6. Les étrangers reconnus "réfugiés" bénéficient de plein droit d'une carte de résident de 10 ans, renouvelable automatiquement, qui vaut double titre, de séjour et de travail. Elle permet au titulaire de circuler librement sur le territoire français et d'exercer un travail, et donne droit à la protection sociale, prestations sociales et familiales, au même titre qu'un Français. À leur arrivée en France, les réfugiés bénéficient d'un accueil en CADA(Centre d'accueil pour demandeurs d'asile) ou en $\mathrm{CPH}$ (Centre provisoire d'hébergement) dont la mission, outre le logement, est de préparer les personnes par un suivi personnalisé à leur installation et leur insertion sociale et professionnelle en France : informations et aides aux démarches administratives, aide à l'accès à un logement indépendant, à l'emploi et à la formation ...). Un suivi en santé physique et psychique leur est assuré. Ils sont affiliés de droit à la CMU. Les réfugiés doivent renoncer à retourner dans leur pays d'origine. Ils peuvent demander à acquérir la nationalité française. http://www.forumrefugies.org/fr/content/view/full/1909

7. Morillon Anne, "Les réfugiés politiques face à la naturalisation", Hommes et Migrations, $\mathrm{n}^{\circ}$ 1234, 2001, pp. 50-57.

8. La première anticipe les difficultés locales et son intégration professionnelle est facilitée par la présence dans le pays d'accueil de ressources économiques et humaines (familiales) ; la seconde immigre dans des conditions dramatiques, et son intégration professionnelle est rendue difficile par l'absence de qualification professionnelle, les problèmes linguistiques et le manque de relais familial.

9. Rafaï Kadija, Mantovani Jean, Duchier Jenny, Gayral-Taminh Martine, Le vieillissement des migrants. Situation en Midi-Pyrénées, rapport ORSMIP, octobre 2007.

10. Vidal Mai Lan, "L'immigration à la vietnamienne", Esprit, mai?1997, pp. 171-178.

11. Vidal Mai Lan, "L'immigration à la vietnamienne", op. cit.

12. Le Huu Khoa, L'immigration confucéenne en France. On s'exile toujours avec ses ancêtres, Paris, l'Harmattan, 1996.

13. "Les migrants de la première génération gardent les valeurs et coutumes de leur pays d'origine, [...] alors que dès la seconde génération l'assimilation se fait". En l'absence d'apports démographiques dans la durée du pays d'origine, "la seconde génération ne peut que s'assimiler", Fourgeau Catherine, "L'insertion en France des communautés asiatiques", op. cit.

14. Vidal Mai Lan, "L'immigration à la vietnamienne", op. cit.

\section{RÉSUMÉS}

Dès la fin des années soixante-dix, plusieurs centaines de milliers de Vietnamiens décident de fuir leur pays où le régime communiste mène une politique de nationalisation des entreprises et de collectivisation des terres. Pour échapper à cette pression économique et au manque de 
liberté, certains tentent de reconstruire leur vie en France. Ce faisant, ces travailleurs tenaces et indépendants cherchent moins un refuge qu'un lieu où accomplir leur quête de réussite sociale. Parfois au prix d'une rupture avec leur pays d'origine.

\section{AUTEUR}

MARTINE GAYRAL-TAMINH

Anthropologue, INSERM 